\title{
Screening for Intestinal Carriage of Extended-spectrum Beta-lactamase-producing Enterobacteriaceae in Critically Ill Patients: Expected Benefits and Evidence-based Controversies
}

Jean-Ralph Zahar, ${ }^{1,2}$ Stijn Blot, ${ }^{3,4}$ Patrice Nordmann, ${ }^{5,6,7}$ Romain Martischang, ${ }^{8}$ Jean-François Timsit, ${ }^{2,9}$ Stephan Harbarth, ${ }^{8}$ and François Barbier ${ }^{10}$

${ }^{1}$ Infection Control Unit, Avicenne University Hospital, Assistance Publique - Hôpitaux de Paris, Bobigny, and ${ }^{2}$ INSERM, Infection Antimicrobial Modelling Evolution, UMR 1137 , Paris Diderot, Sorbonne Paris Cité University, France; ${ }^{3}$ Department of Internal Medicine, Ghent University, Belgium; ${ }^{4}$ Burns, Trauma and Critical Care Research Centre, Centre for Clinical Research, Faculty of Medicine, University of Queensland, Brisbane, Australia; ${ }^{5}$ Medical and Molecular Microbiology Unit, Department of Medicine, Faculty of Science, INSERM European Unit and ${ }^{6}$ Swiss National Reference Center for Emerging Antibiotic Resistance (NARA), University of Fribourg, ${ }^{7}$ Institute for Microbiology, University of Lausanne and University Hospital Centre, and ${ }^{8}$ Infection Control Program and World Health Organization Collaborating Center, Division of Infectious Diseases, Geneva University Hospitals and Faculty of Medicine, Switzerland; ${ }^{9}$ Medical and Infectious Diseases Intensive Care Unit, Bichat-Claude Bernard Hospital, APHP, Paris, and ${ }^{10}$ Medical Intensive Care Unit, La Source Hospital, Orléans, France

The rising burden of intensive care unit (ICU)-acquired infections due to extended-spectrum beta-lactamase-producing Enterobacteriaceae (ESBL-E) strengthens the requirement for efficient prevention strategies. The detection of intestinal carriage of ESBL-E through active surveillance cultures (ASC) and the implementation of contact precautions (CP) in carriers are currently advocated in most high-income countries, to prevent cross-transmission and subsequent ESBL-E infections in critically-ill patients. Yet, recent studies have challenged the benefit of ASC and CP in controlling the spread of ESBL-E in ICUs with high compliance to standard hygiene precautions and no ongoing outbreak of ESBL-producing Klebsiella pneumoniae or Enterobacter spp. Besides, given their debated performance to positively predict which patients are at risk of ESBL-E infections, ASC results appear of limited value to rationalize the empirical use of carbapenems in the ICU, emphasizing the urgent need for novel anticipatory and diagnostic approaches. This Viewpoint article summarizes the available evidence on these issues.

Keywords. extended-spectrum beta-lactamase; contact precautions; critical care; carbapenem; ventilator-associated pneumonia.

The rising prevalence of intensive care unit (ICU)-acquired infections due to extended-spectrum beta-lactamase-producing Enterobacteriaceae (ESBL-E) and the human and economic costs that they induce intensify the necessity for efficient prevention strategies [1]. Since the gut microbiome stands as the main reservoir of invasive ESBL-E strains, a search-and-isolate strategy, based on screening for intestinal carriage through active surveillance cultures (ASC) and the implementation of contact precautions (CP) in identified carriers, is currently advocated by international guidelines for ICUs facing ESBL-E endemicity or ongoing outbreaks $[2,3]$. This policy primarily aims at preventing the cross-transmission of strains and/ or ESBL-encoding plasmids and, thereby, subsequent ESBL-E infections in patients not colonized at admission. In addition, since prior colonization acts as a strong risk factor for ESBL-E infection, ASC results could assist the fine-tuning of

Correspondence: F. Barbier, Medical Intensive Care Unit, La Source Hospital, CHR Orléans, 14 Hospital Ave, 45067-Orléans, France (francois.barbier@chr-orleans.fr). empirical antibiotic therapy in critically-ill patients with sepsis by inciting intensivists to opt for carbapenems rather than other broad-spectrum beta-lactams in documented ESBL-E carriers $[1,4]$. However, the benefit of ASC for controlling the spread of ESBL-E and rationalizing the use of carbapenems in the ICU has been increasingly challenged by the recent literature. In this short narrative review, we sought to summarize the available evidence on these issues.

\section{ACTIVE SURVEILLANCE CULTURES AND CONTACT PRECAUTIONS IN THE CRITICAL CARE ENVIRONMENT}

ESBL-E-notably, CTX-M-producing Escherichia coli-have globally disseminated in the community, with estimated carriage prevalences ranging from 2 to $12 \%$ in Europe, 5 to $47 \%$ in Africa, 7 to $44 \%$ in Southeast Asia, and 29 to $63 \%$ in the West Pacific area [5]. This pandemic drives a continuous influx of ESBL-E into the hospital system that adds to the pool of inpatients colonized with healthcare-associated lineages. Thus, carriage prevalence at ICU admission is rapidly increasing, and now commonly reaches $10-15 \%$ in Europe and up to $40 \%$ in certain Asian countries [6], although marked fluctuations are observed depending on the hospital location and case mix (Supplementary Table S1). Of note, the prevalence of imported 
carriage in a given ICU has been linked to the likelihood of ESBL-E acquisition among patients not colonized at admission, a phenomenon referred as to colonization pressure [7, 8]. This, along with variations in infection control policies, may explain why the average in-ICU acquisition rates remain quite limited in Europe and the Americas (ie, 3-4\%) while exceeding 20\% in high-prevalence areas [9] (Supplementary Table S1).

In addition to hand hygiene $(\mathrm{HH})$ and other standard precautions, the concept of CP implies the utilization of single-bed rooms; patient-dedicated equipment; single-use gloves and gowns for healthcare workers during contacts with carriers or contaminated environments; and the signalization of carriage to ensure compliance [3]. Historically, CP have proven efficiency to control ICU outbreaks of hospital-associated ESBL-E clones, especially ESBL-producing Klebsiella pneumoniae and Enterobacter spp, both being associated with a more pronounced ability for patient-to-patient spreading than ESBLproducing E. coli $[10,11]$. The determinants of this observation are manifold. One may suggest that patients colonized with ESBL-producing K. pneumoniae or Enterobacter spp. differ from those carrying ESBL-producing E. coli in terms of comorbidities and prior contacts with the healthcare system [12, 13]. This could reflect more requirements for nursing care and invasive procedures, as well as more frequent antimicrobial exposure (that is, a more severe intestinal dysbiosis, with a higher faecal relative abundance of ESBL-E), resulting in an increase in the probability of cross-transmission events. Also, prolonged environmental contamination is probably less of an issue with ESBL-producing E. coli than with other ESBL-E.

Yet, apart from epidemic situations, controversies have emerged on whether a systematic ASC and CP policy may help in preventing ESBL-E cross-transmission in hospital units applying current hygiene standards. Studies conducted in wards notably failed to demonstrate a superiority of CP to contain the spread of ESBL-producing E. coli, when compared to standard precautions [14]. It has also been shown that ESBL-E cross-transmission occurs rarely-even in double-bed roomsin wards with a high level of compliance to standard precautions $[10,15]$. Similar results have been increasingly reported in the critical care setting over the recent years. In the European multi-ICU MOSAR trial, universal screening for the carriage of multidrug-resistant (MDR) Enterobacteriaceae (mostly non-E. coli ESBL-E) and the implementation of CP in carriers had no measurable added value on acquisition rates after a first educational phase that increased $\mathrm{HH}$ compliance from 52 to $77 \%$ and included daily chlorhexidine body-washing [16]. The MOSAR investigators confirmed afterwards that the transmissibility of ESBL-E between ICU patients was actually weak, and was even 3 times lower for ESBL-producing E. coli when compared to other ESBL-E (number of secondary cases per index carrier: 0.047 and 0.17 , respectively) [17]. These findings corroborate those of several single-center studies based on molecular methods (strain and/or ESBL typing) that reported very low rates of cross-transmission from documented ESBL-E carriers, including in units without single-bed rooms, suggesting other potential sources of acquisition, such as healthcare workers, contaminated environmental reservoirs, or patient transports outside the ICU $[18,19]$.

Contact isolation measures have been associated with a variety of deleterious side-effects, including patients' psychological distress and an increased hazard of adverse events or medical errors $[20,21]$. Another key issue is the relatively high frequency of false-negative ASC samples-up to $25 \%-$ due to incorrect rectal swabbing procedures or colonization densities of ESBL-E below the detection thresholds [10]. Next, a policy of universal ASC generates both a massive workload for laboratory staffs and substantial expenditures for the hospital system, although its health-economic benefit has not been re-appraised in the contemporary epidemiological context. However, in a dynamic model of ESBL-E dissemination in a 10-bed ICU with an assumed baseline acquisition rate of $15 \%$, improving $\mathrm{HH}$ compliance before and after each contact with patients from $60 \%$ to $80 \%$ was more effective and cost saving than routine carriage screening and CP isolation [22].

The targeted screening of patients with pre-specified risk factors for ESBL-E carriage (ie, recent antimicrobial exposure and/or transfer from long-term care facilities, wards, or other ICUs, especially if abroad) appears as a potentially cost-saving alternative to extended ASC [23]. In 2 before-and-after studies conducted in French ICUs with rates of imported ESBL-E carriage in the range of those usually reported in Northern European countries (that is, 6-7\%), switching from universal to targeted ASC had no impact on the incidence of ICU-acquired ESBL-E infections [24, 25]. Of note, patients admitted directly from home through the emergency department and without known predisposing factors for ESBL-E carriage may be colonized by community-acquired ESBL-producing E. coli. These patients would be missed by a policy of targeted screening; nonetheless, and as discussed above, the hazard of cross-transmission from such unidentified carriers of ESBL-producing E. coli is probably negligible, provided that standard precautions are strictly applied. More pragmatically, in 2 recent studies conducted in ICUs with a relatively low prevalence of ESBL-E, high compliance to standard precautions, and only single-bed rooms, no change was observed in the incidence densities of ICU-acquired ESBL-E colonization and infection following the complete discontinuation of ASC and CP [26, 27], further raising doubt regarding the relevance of these measures in the absence of an outbreak. Still, ASC may be warranted for transferred or repatriated patients at high risk of carriage of carbapenemase-producing Enterobacteriaceae or other extensively drug-resistant Gram-negative bacteria (GNB), such as pan-resistant Acinetobacter spp. 


\section{ACTIVE SURVEILLANCE CULTURES DATA AND EMPIRICAL ANTIMICROBIALTHERAPY}

ASC may provide relevant ecological insights at both individual and ICU levels for the steering of empirical therapy in critically-ill patients. Yet, the relative risk of ICU-acquired ESBL-E infection is up to 50-fold higher in patients with a previously-documented ESBL-E carriage than in those without [9]. Considering that the impaired outcome associated with ESBL-E infections mainly ensues from ineffective first-line coverage [28], the colonization status is understandably seen as a decisional tool to optimize the likelihood of adequate empirical therapy in patients with suspected nosocomial sepsis [1]. Nevertheless, this approach fosters a massive over-consumption of carbapenems in carriers not infected with ESBL-E [4], which may then enhance the spread of non-fermenting GNB exhibiting intrinsic (eg, Stenotrophomonas maltophilia) or acquired (eg, Pseudomonas aeruginosa mutants with modified OprD porin) resistance to this antimicrobial class [29]. This excess use of carbapenems could also contribute to the ongoing pandemic of carbapenemase-producing Enterobacteriaceae [30], although definite evidence is still lacking to confirm this hypothesis.

Overall, ESBL-E infections occur during ICU stays in only 10 to $25 \%$ of critically-ill patients with intestinal colonization $[4,6,26,31]$. In those receiving mechanical ventilation, ESBL-E are responsible for $\sim 40 \%$ of ventilator-associated pneumonia (VAP) [6, 32], while accounting for merely $7 \%$ of infection-related ventilator-associated complications [33]. Therefore, in an era of growing prevalence of colonization, identifying those carriers at risk for infection constitutes a pivotal challenge for carbapenem sparing in the ICU. Studies that focused on this issue yielded conflicting results on the predictive role of clinical parameters, such as prior length of the ICU stay, previous exposure to non-carbapenem antimicrobials (including beta-lactam/beta-lactamase inhibitor combinations, third-generation cephalosporins, or fluoroquinolones), or imported versus ICU-acquired carriage [6, 31, 33]. Patients colonized with ESBL-producing K. pneumoniae or Enterobacter spp. are seemingly at higher risk of infection than those colonized with ESBLproducing E. coli $[6,11]$, an association that might depend on clinical features of the carriers rather than on differences in invasiveness between species. To date, except for the protective effect of a recent exposure to carbapenems, no reliable predictor of ESBL-E infection may help limiting their empirical use in documented carriers [33]. Moreover, if the absence of documented colonization has a $>90 \%$ negative predictive value for ESBL-E infections [34], it does not definitively rule out the involvement of such pathogens, due to the aforementioned possibility of a false-negative ASC or, more anecdotally, an acquisition of carriage between the last available ASC sample and the occurrence of infection. Also, a negative ASC sample for ESBL-E does not exclude an infection caused by other carbapenem-requiring GNB (eg, ceftazidime-resistant $P$. aeruginosa or
MDR Acinetobacter baumannii). It is especially noteworthy that roughly half of culture-positive VAP episodes in ESBL-E carriers implicate non-fermenting GNB (including carbapenem-resistant isolates), alone or in combination with ESBL-E [6, 32, 33]. Hence, a policy of routine screening for intestinal carriage of ESBL-E appears of limited value to rationalize the use of carbapenems in ICU patients.

\section{DEALING WITHTHE EXTENDED-SPECTRUM BETA- LACTAMASE-PRODUCING ENTEROBACTERIACEAE PANDEMIC: PERSPECTIVES FOR INTENSIVISTS}

The prevalence of ESBL-E carriage at ICU admission and the resulting colonization pressure are expected to increase steadily in the years to come, unless strong counter-measures are taken. In this global endemic situation, universal interventions could more effectively prevent cross-transmission than a carrier-centred approach. Sustained efforts to ensure a high level of compliance to $\mathrm{HH}$ (that is, above $80 \%$ ) and other standard measures (eg, environmental disinfection and handling of excreta) are of paramount importance [35]. Overall, routine ASC and the implementation of CP should now be focused on highly-resistant pathogens, such as vancomycin-resistant enterococci or carbapenemase-producing GNB.

Antimicrobial stewardship initiatives are equally essential, since avoiding agents that degrade the normal gut microbiome and the colonization resistance that it confers might protect against ESBL-E acquisition [1,35]. It has notably been reported that prior exposure to beta-lactam/beta-lactamase inhibitor combinations, third-generation cephalosporins (3GC), fluoroquinolones, and even carbapenems may predispose patients to in-ICU acquisition of ESBL-E carriage [8, 9]. Along this line, comparative metagenomic-based studies are warranted to better appraise the impact of antimicrobials on the gut ecosystem, including for agents without activity against Enterobacteriaceae but with a potent anti-anaerobe effect (eg, metronidazole) [36]. Antimicrobial adsorption in the intestinal lumen represents another promising track to reduce their ecological side effects [37].

New algorithms to restrain the empirical use of carbapenems in patients at risk for ESBL-E infection should be considered, given the poor specificity of qualitative ASC. Features of ESBL-E strains (especially virulence determinants) and those of the carrier's gut microbiome (namely, richness and diversity) could putatively impact the hazard of infection [36]. Next-generation sequencing technologies may yield new insights on the virulome and invasiveness of a given ESBL-E strain-as well as its potential for patient-to-patient dissemination - although such markers will not be shortly available at a bedside [38]. Also, the faecal relative abundance of MDR pathogens-including ESBL-E_correlates with the likelihood of infection in non-ICU patients [39-41], suggesting a potential role for quantitative ASC in critically-ill carriers. Then, in mechanically-ventilated patients, oropharyngeal and lower respiratory tract surveillance cultures have been shown 
to usefully predict the pathogens responsible for subsequent VAP $[42,43]$; however, this point remains to be specifically addressed for ESBL-E VAP. Next, recently-released beta-lactam/betalactamase inhibitor combinations (ie, ceftolozane-tazobactam and ceftazidime-avibactam) exhibit activity against multidrugresistant $P$. aeruginosa (including carbapenem-resistant isolates), as well as certain ESBL-E strains (particularly ESBL-producing E. coli) [44], and could theoretically be considered as empirical regimen when both pathogens are suspected. However, clinical and ecological data remain somewhat scarce in critically-ill patients [45], leaving space for further studies to define their potential role as first-line drugs.

On a short-term basis, rapid diagnostic tools stand as the most pragmatic option to detect or exclude an ESBL-E in clinical samples and customize the empirical regimen of ICU patients when an infectious event arises, or to allow earlier de-escalation in those initially treated with a carbapenem. These tests may first be based on chromogenic assays that rapidly detect 3GC-hydrolyzing enzymes (including ESBL) on clinical specimens or early cultures. Direct antimicrobial susceptibility testing (AST) on clinical samples (eg, broncho-alveolar lavage fluid for patients with suspected VAP) is another relevant approach, as it provides susceptibility data from 24 to 48 hours earlier than conventional, subculture-based AST. Moreover, several molecular assays, allowing the detection of ESBL-encoding genes on clinical samples, have been recently released. Although their accuracy still requires in-depth and multi-center appraisal in ICU patients, these tools raise major hopes for the improvement of antibiotic stewardship practices in this specific population. Table 1 summarizes the available evidence on the diagnostic performances and potential applications for these tests.

\section{CONCLUSIONS}

In an era of global ESBL-E dissemination and a massive influx of colonized patients in the ICU setting, we believe that infection prevention strategies should focus on universal measures-with the aim of ensuring a high level of compliance to $\mathrm{HH}$ and other standard precautions - and not on a search-and-isolate policy based on the detection of all ESBL-E carriers through intestinal ASC (Table 2). Targeted screening and CP remain a conceivable

Table 1. Selection of Commercially Available or Homemade Diagnostic Tests for Earlier Detection of Extended-spectrum Beta-lactamase-producing Enterobacteriaceae in Clinical Samples

\begin{tabular}{|c|c|c|c|c|}
\hline Diagnostic Tests & Clinical Samples & $\begin{array}{c}\text { Performance for ESBL-E } \\
\text { Detection (Available Published Data) }\end{array}$ & $\begin{array}{l}\text { Time From } \\
\text { Sampling to Results }\end{array}$ & $\begin{array}{l}\text { Selected } \\
\text { References }\end{array}$ \\
\hline $\begin{array}{l}\beta \text {-Lacta Test } \\
\text { (Bio-Rad, France), }^{\text {a }} \\
\text { chromogenic tests for the } \\
\text { detection of } 3 \mathrm{GC} \text {-hydrolyzing } \\
\text { enzymes (including ESBL) }\end{array}$ & $\begin{array}{l}\text { Urine with GNB on DE; } \\
\text { tracheal aspirate (MV patients) } \\
\text { with GNB on DE and/or } \\
\text { culture } \geq 10.4 \mathrm{cfu} / \mathrm{mL} \text {; } \\
\text { blood culture positive for GNB }\end{array}$ & $\begin{array}{l}\text { Se } 87-100 \% \text {, Sp } 100 \% \text {; Se } 100 \% \text {, Sp } \\
\quad 100 \% \text {, PPV } 100 \% \text {, NPV 100\%; } \\
\text { Se } 100 \% \text {, Sp } 96 \% \text {, PPV } 90 \% \text {, NPV } 100 \%\end{array}$ & 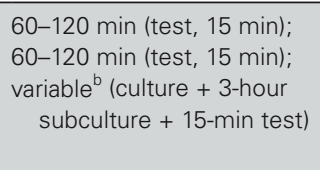 & $\begin{array}{l}{[46]} \\
{[47]} \\
{[48]}\end{array}$ \\
\hline $\begin{array}{l}\text { Rapid ESBL NP Test }^{\mathbf{c}} \\
\text { (homemade), } \\
\text { chromogenic tests for the } \\
\text { detection of 3GC-hydrolyzing } \\
\text { enzymes (including ESBL) }\end{array}$ & $\begin{array}{l}\text { Urine with GNB on DE; } \\
\text { blood culture positive for GNB }\end{array}$ & $\begin{array}{l}\text { Se } 100 \% \text {, Sp } 99 \% \text {, PPV } 98 \% \text {, NPV } 99 \% \\
\text { Se } 100 \% \text {, Sp } 100 \% \text {, PPV } 100 \% \text {, NPV } \\
\quad 100 \%\end{array}$ & $\begin{array}{l}60-120 \text { min (test, } 15 \mathrm{~min}) \\
\text { Variable }^{\text {b }} \text { (test, 30-45 min) }\end{array}$ & $\begin{array}{l}{[49]} \\
{[50,51]}\end{array}$ \\
\hline $\begin{array}{l}\text { Direct AST on respiratory } \\
\text { sample without sub-culture } \\
\text { (homemade) }\end{array}$ & $\begin{array}{l}\text { Broncho-alveolar lavage (MV } \\
\text { patients) with GNB on DE }\end{array}$ & $\begin{array}{l}\text { Se } 100 \% \text {, Sp 95\%, PPV 94\%, NPV } 100 \% \\
\text { (values are for } 3 \mathrm{GC} \text { resistance in } \\
\text { Enterobacteriaceae: ESBL and other } \\
\text { mechanisms) }\end{array}$ & 18-24 hours & [52] \\
\hline $\begin{array}{l}\text { Verigene BC-GN } \\
\text { (Luminex), automated } \\
\text { multiplex PCR for pathogen } \\
\text { identification and detection of } \\
\text { bla }_{\text {CTX-M }} \text { genes }\end{array}$ & Blood culture positive for GNB & $\begin{array}{l}\text { Se } 80-93 \%, \text { Sp 99-100\%, NPV } \\
\text { 93-99\%, PPV 97-100\% (values are } \\
\text { for Escherichia coli and Klebsiella } \\
\text { pneumonia only) }\end{array}$ & Variable $^{\text {b }}$ (test, 2.5 hours) & {$[53,54]$} \\
\hline $\begin{array}{l}\text { Unyvero } \\
\text { (Curetis, Germany) } \\
\text { Automated multiplex PCR for } \\
\text { pathogen identification and } \\
\text { detection of bla } a_{\text {CTX-M }} \text { genes }\end{array}$ & $\begin{array}{l}\text { Tacheal aspirate and broncho- } \\
\text { alveolar lavage (HPN } \\
\text { cartridge); blood culture } \\
\text { (BCU cartridge); } \\
\text { urine (UTI cartridge) }\end{array}$ & $\begin{array}{l}\text { Se } 100 \% \text {, Sp } 85-95 \% \text {, NPV } 100 \% \text {, PPV } \\
20-40 \% \text { (values are for E. coli and } \\
\text { K. pneumonia only); } \\
\text { not published; } \\
\text { not published }\end{array}$ & $\begin{array}{l}4-5 \text { hours; } \\
4-5 \text { hours; } \\
4-5 \text { hours }\end{array}$ & $\begin{array}{l}{[55]} \\
\cdots \\
\cdots\end{array}$ \\
\hline $\begin{array}{l}\text { Accelerate pheno system } \\
\text { (Accelerate Diagnostics) } \\
\text { Automated FISH (pathogen } \\
\text { identification) and AST }\end{array}$ & Positive blood culture & $\begin{array}{l}\text { Agreement with culture-based } \\
\text { AST for ceftriaxone resistance in } \\
\text { Enterobacteriaceae, } 95-97 \%\end{array}$ & Variable $^{\mathrm{b}}$ (test, 6-7 hours) & {$[56,57]$} \\
\hline
\end{tabular}

Abbreviations: $3 G C$, third-generation cephalosporins; AST, antimicrobial susceptibility testing; DE, direct examination; ESBL, extended-spectrum beta-lactamase; ESBLE, ESBL-producing Enterobacteriaceae; FISH, fluorescence in situ hybridization; GNB, Gram-negative bacteria; MV, mechanical ventilation; NPV, negative predictive value; PCR, polymerase chain reaction; PPV positive predictive value; Se, sensibility; Sp, specificity.

${ }^{a}$ The $\beta$-Lacta Test detects all cephalosporin-hydrolyzing enzymes, including ESBL; chromosomal and plasmid-borne AmpC cephalosporinases; and carbapenemases, which may decrease the specificity of this test for the detection of ESBL in settings where other cephalosporine-hydrolyzing beta-lactamases are prevalent.

${ }^{\text {b}}$ Depending on the time to positivity of blood cultures (usually 6 to 12 hours for bloodstream infections, due to Enterobacteriaceae).

${ }^{\mathrm{T}}$ The Rapid ESBL NPTest only detects ESBL. 
- Rates of imported ESBL-E carriage are rapidly increasing in most ICUs worldwide, owing to the successful spread of these pathogens (especially CTX-Mproducing $E$. coll) in both community and hospital ecosystems.

- Universal screening for intestinal carriage of ESBL-E through ASC and the implementation of contact precautions in identified carriers appears of limited added value to prevent cross-transmission events in those ICUs with high compliance to standard precautions and no ongoing outbreak of ESBL-producing K. pneumoniae or Enterobacter spp.

- The results of qualitative ASC for ESBLE carriage are neither sufficient nor efficient to rationalize the empirical use of carbapenems in ICU patients.

- Novel predictive and diagnostic approaches (including phenotypic or molecular rapid diagnostic tools on clinical samples) are needed to customize the empirical antimicrobial therapy in critically-ill patients at risk for ICU-acquired ESBL-E infections.

Abbreviations: ASC, active surveillance cultures; ESBL, extended-spectrum beta-lactamase; ESBL-E, ESBL-producing Enterobacteriaceae; ICU, intensive care unit.

approach in critical-care environments with uncontrolled endemicity or outbreaks of ESBL-producing K. pneumoniae or Enterobacter spp., while the available evidence argues against the usefulness of such interventions in ICUs where ESBLproducing E. coli predominate. Given the rising incidence of healthcare-associated infections due to carbapenem-resistant GNB, a paradigm shift from ACS-based empirical regimen to new diagnostic approaches is urgently needed to restrain the overconsumption of carbapenems in patients at risk for ICUacquired ESBL-E infections. The use of clinical algorithms, including rapid diagnostic tests, which are able to rule out the involvement of ESBL-E based on high negative predictive values, will probably help ICU physicians to choose the best therapeutic options in patients with suspected healthcare-associated infections.

\section{Supplementary Data}

Supplementary materials are available at Clinical Infectious Diseases online. Consisting of data provided by the authors to benefit the reader, the posted materials are not copyedited and are the sole responsibility of the authors, so questions or comments should be addressed to the corresponding author.

\section{Notes}

Financial support. This work was supported by the Swiss National Science Foundation (grant 4049-40-106294/1 for an ongoing clinical trial entitled "An interventional study to evaluate the impact of a rapid screening strategy in improving nosocomial ESBL-E and CPE control in critically ill patients").

Potential conflicts of interest. J.-R. Z. has received consulting fees, speaker fees, and conference invitations from Merck, Sharp and Dohme (MSD); conference invitations from Pfizer; and consulting fees from Sanofi. P. N. has received consulting fees, speaker fees, and conference invitations from MSD, Shionogi, and Pfizer. J.-F. T. has received consulting and lecture fees from MSD; consulting fees from Abbott, MaaT Pharma, and Icuresearch; and lecture fees from Pfizer and Biomerieux. J.-F. T.s university hospital also received research grants from 3M, Pfizer, MSD, and Biomerieux. S. H. has received funding from the European Commission and the Swiss National Science Foundation for several clinical studies related to extended-spectrum ESBL-E, and has received consulting fees from Sandoz, Bayer, and DNA Electronics. F. B. has received consulting fees, speaker fees, and conference invitations from MSD and conference invitations from Pfizer. All other authors report no potential conflicts. All authors have submitted the ICMJE Form for Disclosure of Potential Conflicts of Interest. Conflicts that the editors consider relevant to the content of the manuscript have been disclosed.

\section{References}

1. Bassetti M, De Waele JJ, Eggimann P, et al. Preventive and therapeutic strategies in critically ill patients with highly resistant bacteria. Intensive Care Med 2015; 41:776-95.

2. Centers for Disease Control and Prevention Healthcare Infection Control Practices Advisory Committee. Management of multidrug-resistant organisms in healthcare settings. Available at: https://www.cdc.gov/infectioncontrol/guidelines/mdro. Accessed 1 May 2018.

3. Tacconelli E, Cataldo MA, Dancer SJ, et al.; European Society of Clinical Microbiology. ESCMID guidelines for the management of the infection control measures to reduce transmission of multidrug-resistant Gram-negative bacteria in hospitalized patients. Clin Microbiol Infect 2014; 20(Suppl 1):1-55.

4. Barbier F, Pommier C, Essaied W, et al.; OUTCOMEREA Study Group. Colonization and infection with extended-spectrum $\beta$-lactamase-producing Enterobacteriaceae in ICU patients: what impact on outcomes and carbapenem exposure? J Antimicrob Chemother 2016; 71:1088-97.

5. Karanika S, Karantanos T, Arvanitis M, Grigoras C, Mylonakis E. Fecal colonization with extended-spectrum beta-lactamase-producing Enterobacteriaceae and risk factors among healthy individuals: A systematic review and metaanalysis. Clin Infect Dis 2016; 63:310-8.

6. Razazi K, Mekontso Dessap A, Carteaux G, et al. Frequency, associated factors and outcome of multi-drug-resistant intensive care unit-acquired pneumonia among patients colonized with extended-spectrum $\beta$-lactamase-producing Enterobacteriaceae. Ann Intensive Care 2017; 7:61.

7. Masse J, Elkalioubie A, Blazejewski C, et al. Colonization pressure as a risk factor of ICU-acquired multidrug resistant bacteria: a prospective observational study. Eur J Clin Microbiol Infect Dis 2017; 36:797-805.

8. Razazi K, Derde LP, Verachten M, Legrand P, Lesprit P, Brun-Buisson C. Clinical impact and risk factors for colonization with extended-spectrum $\beta$-lactamase-producing bacteria in the intensive care unit. Intensive Care Med 2012; 38:1769-78.

9. Detsis M, Karanika S, Mylonakis E. ICU acquisition rate, risk factors, and clinical significance of digestive tract colonization with extended-spectrum beta-lactamase-producing Enterobacteriaceae: a systematic review and meta-analysis. Crit Care Med 2017; 45:705-14.

10. Tschudin-Sutter S, Lucet JC, Mutters NT, Tacconelli E, Zahar JR, Harbarth S. Contact precautions for preventing nosocomial transmission of extended-spectrum $\beta$ lactamase-producing Escherichia coli: A point/counterpoint review. Clin Infect Dis 2017; 65:342-7.

11. Gorrie CL, Mirceta M, Wick RR, et al. Gastrointestinal carriage is a major reservoir of Klebsiella pneumoniae infection in intensive care patients. Clin Infect Dis 2017; 65:208-15.

12. Scheuerman O, Schechner V, Carmeli Y, et al.; REIPI/ESGBIS/INCREMENT investigators. Comparison of predictors and mortality between bloodstream infections caused by ESBL-producing Escherichia coli and ESBL-producing Klebsiella pneumoniae. Infect Control Hosp Epidemiol 2018; 39:660-7.

13. Freeman JT, Rubin J, McAuliffe GN, et al. Differences in risk-factor profiles between patients with ESBL-producing Escherichia coli and Klebsiella pneumoniae: a multicentre case-case comparison study. Antimicrob Resist Infect Control 2014; 3:27.

14. Zahar JR, Poirel L, Dupont C, Fortineau N, Nassif X, Nordmann P. About the usefulness of contact precautions for carriers of extended-spectrum beta-lactamase-producing Escherichia coli. BMC Infect Dis 2015; 15:512.

15. Tschudin-Sutter S, Frei R, Dangel M, Stranden A, Widmer AF. Rate of transmission of extended-spectrum beta-lactamase-producing Enterobacteriaceae without contact isolation. Clin Infect Dis 2012; 55:1505-11.

16. Derde LPG, Cooper BS, Goossens H, et al.; MOSAR WP3 Study Team. Interventions to reduce colonisation and transmission of antimicrobial-resistant bacteria in intensive care units: an interrupted time series study and cluster randomised trial. Lancet Infect Dis 2014; 14:31-9. 
17. Gurieva T, Dautzenberg MJD, Gniadkowski M, Derde LPG, Bonten MJM, Bootsma MCJ. The transmissibility of antibiotic-resistant Enterobacteriaceae in intensive care units. Clin Infect Dis 2018; 66:489-93.

18. Repessé X, Artiguenave M, Paktoris-Papine S, et al. Epidemiology of extended-spectrum beta-lactamase-producing Enterobacteriaceae in an intensive care unit with no single rooms. Ann Intensive Care 2017; 7:73.

19. Alves M, Lemire A, Decré D, et al. Extended-spectrum beta-lactamase-producing Enterobacteriaceae in the intensive care unit: acquisition does not mean cross-transmission. BMC Infect Dis 2016; 16:147.

20. Morgan DJ, Diekema DJ, Sepkowitz K, Perencevich EN. Adverse outcomes associated with Contact Precautions: a review of the literature. Am J Infect Contro 2009; 37:85-93.

21. Zahar JR, Garrouste-Orgeas M, Vesin A, et al. Impact of contact isolation for multidrug-resistant organisms on the occurrence of medical errors and adverse events. Intensive Care Med 2013; 39:2153-60.

22. Kardaś-Słoma L, Lucet JC, Perozziello A, et al. Universal or targeted approach to prevent the transmission of extended-spectrum beta-lactamase-producing Enterobacteriaceae in intensive care units: a cost-effectiveness analysis. BMJ Open 2017; 7:e17402.

23. Mutters NT, Günther F, Frank U, Mischnik A. Costs and possible benefits of a two-tier infection control management strategy consisting of active screening for multidrug-resistant organisms and tailored control measures. J Hosp Infect 2016 93:191-6.

24. Dananché C, Bénet T, Allaouchiche B, et al. Targeted screening for third-generation cephalosporin-resistant Enterobacteriaceae carriage among patients admitted to intensive care units: a quasi-experimental study. Crit Care 2015 19:38.

25. Djibré M, Fedun S, Le Guen P, et al. Universal versus targeted additional contact precautions for multidrug-resistant organism carriage for patients admitted to an intensive care unit. Am J Infect Control 2017; 45:728-34.

26. Jalalzaï W, Boutrot M, Guinard J, et al. Cessation of screening for intestinal carriage of extended-spectrum $\beta$-lactamase-producing Enterobacteriaceae in a low-endemicity intensive care unit with universal contact precautions. Clin Microbiol Infect 2018; 24:429.e7-429.e12.

27. Renaudin L, Llorens M, Goetz C, et al. Impact of discontinuing contact precautions for MRSA and ESBLE in an intensive care unit: a prospective noninferiority before and after study. Infect Control Hosp Epidemiol 2017 $38: 1342-50$

28. Rottier WC, Ammerlaan HS, Bonten MJ. Effects of confounders and intermediates on the association of bacteraemia caused by extended-spectrum $\beta$-lactamase-producing Enterobacteriaceae and patient outcome: a meta-analysis. J Antimicrob Chemother 2012; 67:1311-20.

29. Armand-Lefèvre L, Angebault C, Barbier F, et al. Emergence of imipenem-resistant gram-negative bacilli in intestinal flora of intensive care patients. Antimicrob Agents Chemother 2013; 57:1488-95.

30. McLaughlin M, Advincula MR, Malczynski M, Qi C, Bolon M, Scheetz MH. Correlations of antibiotic use and carbapenem resistance in Enterobacteriaceae. Antimicrob Agents Chemother 2013; 57:5131-3.

31. Vodovar D, Marcadé G, Rousseau H, et al. Predictive factors for extended-spectrum beta-lactamase producing Enterobacteriaceae causing infection among intensive care unit patients with prior colonization. Infection 2014 42:743-8.

32. Bruyère $\mathrm{R}$, Vigneron $\mathrm{C}$, Bador $\mathrm{J}$, et al. Significance of prior digestive colonization with extended-spectrum $\beta$-lactamase-producing Enterobacteriaceae in patients with ventilator-associated pneumonia. Crit Care Med 2016; 44:699-706.

33. Barbier F, Bailly S, Schwebel C, et al.; OUTCOMEREA Study Group. Correction to: Infection-related ventilator-associated complications in ICU patients colonised with extended-spectrum $\beta$-lactamase-producing Enterobacteriaceae. Intensive Care Med 2018; 44:1200-2.

34. Carbonne H, Le Dorze M, Bourrel AS, et al. Relation between presence of extended-spectrum $\beta$-lactamase-producing Enterobacteriaceae in systematic recta swabs and respiratory tract specimens in ICU patients. Ann Intensive Care 2017; $7: 13$.

35. Teerawattanapong N, Kengkla K, Dilokthornsakul P, Saokaew S, Apisarnthanarak A, Chaiyakunapruk N. Prevention and control of multidrug-resistant Gramnegative bacteria in adult intensive care units: A systematic review and network meta-analysis. Clin Infect Dis 2017; 64:51-60.

36. Ricard JD, Lisboa T. Caution for chlorhexidine gluconate use for oral care: insufficient data. Intensive Care Med 2018; 44:1162-4.

37. de Gunzburg J, Ghozlane A, Ducher A, et al. Protection of the human gut microbiome from antibiotics. J Infect Dis 2018; 217:628-36.
38. Zhou K, Lokate M, Deurenberg RH, et al. Use of whole-genome sequencing to trace, control and characterize the regional expansion of extended-spectrum $\beta$-lactamase producing ST15 Klebsiella pneumoniae. Sci Rep 2016; 6:20840.

39. Ruppé E, Lixandru B, Cojocaru R, et al. Relative fecal abundance of extended-spectrum- $\beta$-lactamase-producing Escherichia coli strains and their occurrence in urinary tract infections in women. Antimicrob Agents Chemother 2013; 57:4512-7.

40. Woerther PL, Micol JB, Angebault C, et al. Monitoring antibiotic-resistant enterobacteria faecal levels is helpful in predicting antibiotic susceptibility of bacteraemia isolates in patients with haematological malignancies. J Med Microbiol 2015; 64:676-81.

41. Taur Y, Xavier JB, Lipuma L, et al. Intestinal domination and the risk of bacteremia in patients undergoing allogeneic hematopoietic stem cell transplantation. Clin Infect Dis 2012; 55:905-14.

42. Brusselaers N, Labeau S, Vogelaers D, Blot S. Value of lower respiratory tract surveillance cultures to predict bacterial pathogens in ventilator-associated pneumonia: systematic review and diagnostic test accuracy meta-analysis. Intensive Care Med 2013; 39:365-75.

43. Garrouste-Orgeas M, Chevret S, Arlet G, et al. Oropharyngeal or gastric colonization and nosocomial pneumonia in adult intensive care unit patients. A prospective study based on genomic DNA analysis. Am J Respir Crit Care Med 1997; 156:1647-55.

44. Wright $\mathrm{H}$, Bonomo RA, Paterson DL. New agents for the treatment of infections with Gram-negative bacteria: restoring the miracle or false dawn? Clin Microbiol Infect 2017; 23:704-12.

45. Torres A, Zhong N, Pachl J, et al. Ceftazidime-avibactam versus meropenem in nosocomial pneumonia, including ventilator-associated pneumonia (REPROVE): a randomised, double-blind, phase 3 non-inferiority trial. Lancet Infect Dis 2018; 18:285-95.

46. Gallah S, Decré D, Genel N, Arlet G. The $\beta$-Lacta test for direct detection of extended-spectrum- $\beta$-lactamase-producing Enterobacteriaceae in urine. J Clin Microbiol 2014; 52:3792-4.

47. Gallah S, Benzerara Y, Tankovic J, et al. $\beta$ LACTA test performance for detection of extended-spectrum $\beta$-lactamase-producing Gram-negative bacilli directly on bronchial aspirates samples: a validation study. Clin Microbiol Infect 2018; 24:402-8.

48. Compain F, Bensekhri H, Rostane H, Mainardi JL, Lavollay M. $\beta$ LACTA test for rapid detection of Enterobacteriaceae resistant to third-generation cephalosporins from positive blood cultures using briefly incubated solid medium cultures. J Med Microbiol 2015; 64:1256-9.

49. Dortet L, Poirel L, Nordmann P. Rapid detection of extended-spectrum- $\beta$-lactamase-producing Enterobacteriaceae from urine samples by use of the ESBL NDP test. J Clin Microbiol 2014; 52:3701-6.

50. Arca-Suárez J, Galán-Sánchez F, Del Prado Montoro C, Rodríguez-Iglesias MA. A modified ESBL Nordmann/Dortet/Poirel-based protocol to optimize early sepsis management. J Microbiol Methods 2017; 139:45-7.

51. Dortet L, Poirel L, Nordmann P. Rapid detection of ESBL-producing Enterobacteriaceae in blood cultures. Emerg Infect Dis 2015; 21:504-7.

52. Le Dorze M, Gault N, Foucrier A, et al. Performance and impact of a rapid method combining mass spectrometry and direct antimicrobial susceptibility testing on treatment adequacy of patients with ventilator-associated pneumonia. Clin Microbiol Infect 2015; 21:468.e1-6.

53. Pogue JM, Heil EL, Lephart $\mathrm{P}$, et al. An antibiotic stewardship program blueprint for optimizing Verigene BC-GN within an institution. Antimicrob Agents Chemother 2018; 62:e2538-17

54. Siu GK, Chen JH, Ng TK, et al. Performance evaluation of the verigene Grampositive and Gram-negative blood culture test for direct identification of bacteria and their resistance determinants from positive blood cultures in Hong Kong. PLoS One 2015; 10:e0139728.

55. Ozongwu C, Personne Y, Platt G, et al. The Unyvero P55 'sample-in, answerout' pneumonia assay: a performance evaluation. Biomol Detect Quantif 2017; 13:1-6.

56. Pancholi P, Carroll KC, Buchan BW, et al. Multicenter evaluation of the Accelerate Pheno test BC kit for rapid identification and phenotypic antimicrobial susceptibility testing using morphokinetic cellular analysis. J Clin Microbiol 2018; 56:e01329-17

57. Marschal M, Bachmaier J, Autenrieth I, Oberhettinger P, Willmann M, Peter S. Evaluation of the accelerate pheno system for fast identification and anti microbial susceptibility testing from positive blood cultures in bloodstream infections caused by Gram-negative pathogens. J Clin Microbiol 2017; $55: 2116-26$ 